\title{
Dynamic Oligopolistic Games Under Uncertainty: A Stochastic Programming Approach*
}

\author{
Talat Genc ${ }^{\mathrm{a}}$, Stanley S. Reynolds ${ }^{\mathrm{b}}$, and Suvrajeet Sen ${ }^{\mathrm{c}}$
}

September 9, 2005

\begin{abstract}
This paper studies several stochastic programming formulations of dynamic oligopolistic games under uncertainty. We argue that one of the models, namely Games with Probabilistic Scenarios (GPS), provides an appropriate formulation. For such games, we show that symmetric players earn greater expected profits as demand volatility increases. This result suggests that even in an increasingly volatile market, players may have an incentive to participate in the market. The key to our approach is the so-called scenario formulation of stochastic programming. In addition to several modeling insights, we also discuss the application of GPS to the electricity market in Ontario, Canada. The examples presented in this paper illustrate that this approach can address dynamic games that are clearly out of reach for dynamic programming, a common approach in the literature on dynamic games.
\end{abstract}

* This research was carried as part of the first author's dissertation research conducted jointly in Economics and the MORE Institute at the University of Arizona.

a Department of Economics, University of Guelph, Guelph, Ontario, N1G 2W1, Canada tgenc@uoguelph.ca

${ }^{b}$ Department of Economics, Eller College of Management, University of Arizona, Tucson, AZ, 85721 reynolds@eller.arizona.edu

${ }^{c}$ Systems and Industrial Engineering, University of Arizona, Tucson, AZ, 85721 sen@sie.arizona.edu 


\section{Introduction}

We consider stochastic equilibrium problems in which players have a significant stake in technology, and meet their production commitments by investments in a variety of technologies. For example, consider an electric power market consisting of a few players (suppliers), each of whom generates power using a variety of generators. These generators cannot be installed instantaneously, and as a result, investment precedes production by several months, and sometimes, even years. Under these circumstances, players make their investment decisions under uncertainty. The degree of uncertainty may depend on macroeconomic conditions as well as market-specific characteristics. The Stochastic Programming (SP) methodology is based on modeling alternative economic scenarios that may unfold in the future. For the sake of computational tractability, these scenarios are restricted to a finite set, and with each scenario one associates a non-zero probability of occurrence.

The focus of this paper is on the development of models that may be used to predict investment, production, and price trajectories associated with alternative economic scenarios that may unfold. However, these trajectories depend upon the behavior of the players. We will study three alternative behavioral assumptions. In the first formulation, the players make decisions based on collection of probabilistic scenarios, which we refer to as a game with probabilistic scenarios (GPS). Here the trajectories (investment, production, price) will depend on the scenario that unfolds; trajectories will be required to obey a non-clairvoyance condition which states that decisions cannot depend on information revealed in the future. In the SP literature, this condition is also referred to as the non-anticipativity requirement.

The second formulation we investigate is called a game with expected scenarios (GES) where investment decisions are based on an expected scenario (as though the world is deterministic). Once the investment decisions are made in a given period, one of the possible scenarios unfolds, and players make their production decisions in response to the specific scenario that unfolds. This type of behavior is not uncommon in some industries where the inclusion of uncertainty within an investment model leads to a very complicated and sometimes intractable model. For example in the electric power industry, one can invest in a variety of generators (nuclear, hydro, coal, gas etc.) and the 
resulting capacity expansion models can be rather complex (e.g., WASP-IV (2000), Murphy, Sen and Soyster (1982)). Due to the difficulties associated with modeling uncertainty within a complex capacity expansion model, players may decide to replace the probabilistic scenarios by an expected scenario, thus leading to a GES game. Nevertheless, we recognize that since operation (generation) decisions are undertaken when better demand information becomes available (i.e. the scenario unfolds), the production game adapts to the scenario that unfolds. Finally, we study a third formulation which we call a hybrid game $(\mathrm{HG})$ which combines features from the GPS and GES games.

There are a variety of types of non-cooperative equilibria for dynamic games. In this paper we focus on the $\boldsymbol{S}$-adapted open-loop equilibrium. This equilibrium concept was introduced by Zaccour (1987), and Haurie, Zaccour and Smeers (1990). In such an equilibrium each player adopts a strategy that specifies its (production and investment) decisions for each time period and for each possible scenario that can be observed in a time period. In our models, observing a scenario corresponds to knowledge of the current level of demand. A strategy for a firm may be viewed as a contingency plan that specifies actions for each time period and each possible demand state associated with a period. An S-adapted open-loop equilibrium is a set of strategies (plans) for players such that each player's strategy maximizes its expected payoff, given the strategies of the other players. If uncertainty is modeled as a collection of probabilistic scenarios then an equilibrium for a GPS formulation is a S-adapted open loop equilibrium. An excellent overview of the concept of S-adapted equilibrium, and its connections with other concepts and information structures (e.g. stochastic variational inequalities, piecewise open loop information structures etc.) are presented in Haurie and Zaccour (2004).

One of the early applications of the concept of S-adapted equilibrium appears in Haurie et al. (1987), where the European natural gas market problem is used as a basis to predict prices, production and capital investment. In this game theoretic setting producers (USSR, Netherlands, Norway 1, Norway 2, and Algeria) compete in several gas markets (France, Italy, Netherlands, UK, FRGer, BelLux). This data set has also been used by Gurkan, Ozge and Robinson (1999), who use sample-path optimization (simulation based optimization) to solve stochastic variational inequalities. It is important to observe that 
the approach of Gurkan, Ozge and Robinson (1999) is primarily intended for single (rather than multi) period games. At the other end of the spectrum, Haurie and Moresino $(2001,2002)$ have addressed extensions of the notion of S-adapted equilibrium to a continuous-time formulation of Cournot/Nash. More recently, Haurie and Viguier (2003) apply an S-adapted Cournot/Nash equilibrium concept on a stochastic dynamic game of carbon emissions trading among certain countries.

While the equilibrium concept for GPS is based on that proposed in Haurie, Zaccour and Smeers (1990), our development provides extensions of their work in several directions. First, our analysis points to the fact that competing game models such as GES might seem attractive, but using HG, we argue that the GPS game is the most tenable of the three. In addition, we show that under certain assumptions (i.e., symmetric cost structures), the presence of volatility also provides greater expected profits in a game. This provides the intuition about why players in a market may continue to participate, even though market volatility may be on the rise. In addition, we study multistage (sequential) games under uncertainty, and provide a formulation that allows modeling technology additions for applications in which there may be significant lags between the decision to invest, and time at which the plant becomes productive. In contrast to the formulations cited above, we adopt an equivalent scenario-based formulation. The resulting equilibrium conditions are easily applicable for problems with significant lags, and moreover, this formulation is amenable to solution methods for complementarity problems. This helps avoid recursive value function approximations which is the source of the curse of dimensionality in dynamic programming. We illustrate the advantages of this approach with an example that is well out of reach for standard dynamic programming methodology.

We provide conditions for existence and uniqueness of (open-loop) equilibrium for the game formulations that were described above. We also show that players may be worse off in a stochastic game (GPS) than if they play the game deterministically (GES), even though the environment is stochastic. This result distinguishes the multi-player game from a single player setting in which stochastic optimization is known to provide superior expected profits, when compared to deterministic optimization.

The paper is organized as follows. Section 2 introduces the three game formulations 
for the two-stage case and provides an example which illustrates and compares players' performances and optimal decisions in GPS and GES. Section 3 considers multi-stage models of the games and provides two equivalent formulations which can be characterized as either recursive or non-recursive. In addition, we present an application of our methodology to data from the province of Ontario, Canada. This demonstrates the computational power of the SP approach. Section 4 concludes this paper with a discussion of future research.

\section{Some Two-Stage Stochastic Programming Games}

We consider a two-period finite player non-cooperative game where players make investment and production decisions under uncertainty about future demand for the product. In this game, uncertainty can be represented by a finite number of scenarios. Each scenario represents a possible realization of a random process. We assume that players share a common characterization of the random process. In addition, the players know their own production and investment cost functions and their own initial capacities. Each firm strives to maximize their discounted expected payoff. Given the initial capacity levels, all firms make their initial production decisions (in period 0) to maximize current profit and at the same time they choose their investment in production capacity (in period 0 ). These investments in capacity become available for production in the next period (period 1). However demand scenarios for the next period are stochastic, and investment decisions must be made here-and-now. After uncertainty unfolds in period one, players make their production decisions. Since this is a two-stage model, we will not consider any investment opportunities in the final period.

\subsection{A Two Stage Stochastic Game}

Formally this game is defined as follows. Let the players in this game be indexed by $i \in N=\{1,2, \ldots, n\}$, where $n$ is finite. In period 0 , let $K_{i}^{0}=\left(K_{i, 1}^{0}, K_{i, 2}^{0}, \ldots, K_{i, m}^{0}\right)$, where $K_{i, k}^{0}, k=1,2, \ldots, m$, denotes the available capacity at time 0 from technology $k$ for firm $i$. Since the demand is already realized, the players (firms) choose the production quantities $q_{i}^{*}\left(K_{i}^{0}\right)$ for all $i \in N$ such that when $q_{j} \equiv q_{j}^{*}, j \neq i$ then 


$$
q_{i}^{*}\left(K_{i}^{0}\right) \in \arg \max \left\{P\left(Q^{*}\left(K^{0}\right)\right) \sum_{k=1}^{m} q_{i, k}-c_{i}\left(q_{i}\right) \mid q_{i} \in B_{i}\left(K_{i}^{0}\right)\right\}
$$

where $Q^{*}\left(K^{0}\right)=\sum_{i=1}^{n} \sum_{k=1}^{m} q_{i, k}^{*}, q_{i}=\left(q_{i, 1}, q_{i, 2}, \ldots, q_{i, m}\right) . P(Q)$ is the inverse demand function which determines the price of output as a function of total production; this is a strictly decreasing function of total quantity of production in the market. $c_{i}\left(q_{i}\right)$ is the total cost as a function of the vector of outputs for all technologies ${ }^{1}$, and $B_{i}($.$) is a convex$ and compact production set for player $i, B_{i} \subset \mathfrak{R}_{+}^{m}$.

However investment decisions $I_{i}^{*}$ must be chosen at period 0 as well, and this decision must be made in the face of uncertainty which is modeled using a discrete random variable whose outcomes represent possible levels of future demand, indexed by $s$. Let the investment space for player $i$ be denoted as $A_{i}$, which is assumed to be nonempty, and bounded. Thus $I_{i}^{*} \in A_{i}$, such that

$$
I_{i}^{*} \in \arg \max \left\{-F_{i}\left(I_{i}\right)+E\left[f_{i}\left(I_{i}, q_{i}^{*}\left(K_{i}^{1}, \tilde{s}\right), Q^{*}\left(K_{i}^{1},\left\{K_{j}^{1^{*}}\right\}_{j \neq i} ; \tilde{s}\right), \tilde{s}\right] \mid I_{i} \in A_{i} \subseteq \mathfrak{R}_{+}^{m}\right\}\right.
$$

where period 1 production levels are $q_{i}^{*}\left(K_{i}^{1^{*}}, s\right)$ such that,

$$
q_{i}^{*}\left(K_{i}^{1^{*}}, s\right) \in \arg \max \left\{f_{i}\left(I_{i}^{*}, q_{i}, Q^{*}\left(K^{1^{*}} ; s\right), s\right) \mid q_{i} \in B_{i}\left(K_{i}^{1^{*}}\right)\right\} .
$$

Here $\quad Q^{*}\left(K^{1^{*}} ; s\right)=\sum_{i=1}^{n} \sum_{k=1}^{m} q_{i, k}^{*}\left(K_{i}^{1^{*}}, s\right), \quad f_{i}(\cdot)=P\left(Q^{*}\left(K^{1^{*}} ; s\right), s\right) \sum_{k=1}^{m} q_{i, k}-c_{i}\left(q_{i}\right)$, $K_{i}^{1^{*}}=K_{i}^{0}+I_{i}^{*}$. In the above formulation we assume that cost functions $F_{i}($.$) and c_{i}($. are strictly increasing and convex, and $f_{i}($.$) is strictly concave in q_{i}$. In addition, it is assumed that the inverse demand functions for periods zero and one are linear in $Q$, there exists a $Q^{\prime}>0$ such that price is zero for $Q^{\prime} \geq Q$ (for period one, $Q^{\prime}$ depends on the scenario) and the price is finite when the quantity is zero. The strategy space for player $i$, for a given period $t$ denoted as $\Gamma_{i}^{t}$ which is compact and convex and the Cartesian product of the production and investment decisions, so that $\Gamma_{i}^{t}=\mathrm{A}_{i}^{t} \times \mathrm{B}_{i}^{t}$, but for the last period $\Gamma_{i}^{t}=\mathrm{B}_{i}^{t}$, since there is no investment.

\footnotetext{
${ }^{1}$ More generally, the production cost function would also depend on the amount of capital or capacity. This is essentially a short-run cost of production conditional on the amount of the capital input. For an example of this in a dynamic game analysis, see Reynolds (1986).
} 
Given above assumptions and setting there is a unique Nash equilibrium for this two period non-cooperative game (Okuguchi, Szidarovszky (1999)).

\subsection{A Two-Stage Game with Probabilistic Scenarios (GPS)}

The game formulated in (1), (2), and (3) can also be represented by a model using the so-called scenario formulation. Such a formulation provides a convenient mechanism for games in which players face a large number of constraints. Each possible level of period one demand corresponds to one scenario. For a given scenario $s \in S=\{1,2,3, \ldots, \omega\}, \omega<\infty$, player $i$ chooses beginning production quantities as $q_{i}^{*}\left(K_{i, s}^{0}\right)$ such that when $q_{j, s} \equiv q_{j, s}^{*}, j \neq i$ then

$$
q_{i}^{*}\left(K_{i, s}^{0}\right) \in \arg \max \left\{P\left(Q^{*}\left(K_{s}^{0}\right)\right) q_{i, s}-c_{i}\left(q_{i, s}\right) \mid q_{i, s} \in B_{i}\left(K_{i, s}^{0}\right)\right\}
$$

Here $Q^{*}\left(K_{s}^{0}\right)=\sum_{i=1}^{n} \sum_{k=1}^{m} q_{i, k}^{*}\left(K_{i, s, k}^{0}\right), q_{i, s}=\left(q_{i, s, 1}, q_{i, s, 2}, \ldots, q_{i, s, m}\right)$.

For each player $i$ and scenario $s$ investment decisions are made in the first period according to the following.

$$
I_{i, s}^{*} \in \arg \max \left\{-F_{i}\left(I_{i, s}\right)+\left[f_{i}\left(I_{i}, q_{i}^{*}\left(K_{i, s}^{1}, s\right), Q^{*}\left(K_{i, s}^{1},\left\{K_{j, s}^{1^{*}}\right\}_{j \neq i} ; s\right), s\right] \mid I_{i, s} \in A_{i, s} \subseteq \mathfrak{R}_{+}^{m}\right\},\right.
$$

where period 1 production levels are $q_{i}^{*}\left(K_{i, s}^{1^{*}}, s\right)$ which satisfy

$$
q_{i}^{*}\left(K_{i, s}^{1^{*}}, s\right) \in \arg \max \left\{f_{i}\left(I_{i}^{*}, q_{i}, Q^{*}\left(K_{s}^{1^{*}} ; s\right), s\right) \mid q_{i, s} \in B_{i}\left(K_{i, s}^{1^{*}}\right)\right\} .
$$

Again $Q^{*}\left(K_{s}^{1^{*}} ; s\right)=\sum_{i=1}^{n} \sum_{k=1}^{m} q_{i, s, k}^{*}\left(K_{i, s}^{1^{*}}, s\right), \quad f_{i}()=.P\left(Q^{*}\left(K_{s}^{1^{*}} ; s\right), s\right) \sum_{k=1}^{m} q_{i, s, k}-c_{i}\left(q_{i, s}\right)$, $K_{i, s}^{1^{*}}=K_{i, s}^{0}+I_{i, s}^{*}$.

Unfortunately decisions from this formulation cannot be implemented because investment decisions are dependent on future scenarios that are not revealed in the beginning (i.e., period 0). In order to ensure that the decisions can be implemented prior to resolving the uncertainty, we impose the non-anticipativity (non-clairvoyance) constraint as

$$
E\left(I_{i, s}^{*}\right)=I_{i}^{*} \text { for all } i \text { and } s .
$$

This equation means that under uncertainty, planning decisions must be implemented before an outcome of the random variable is observed. For the sake of clarity, we write player $i$ 's objective function and constraints as follows. 
First assume that each player $i$ predicts a market with the following scenarios.

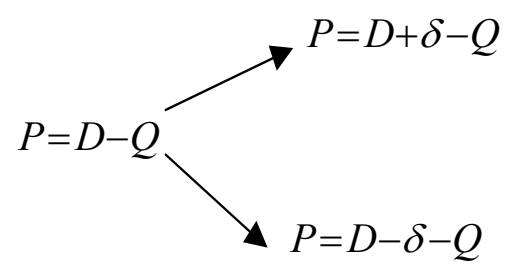

$\mathrm{t}=0 \quad \mathrm{t}=1$

\section{Figure 1: Two-stage demand scenarios}

In the subsequent section we will investigate multi-stage formulations in which a binary tree of this type is used to model the evolution of demand in a wholesale electricity market. Thus a simple scenario tree such as the one shown above may be looked upon as a building block for more realistic models.

For player $i$ the expected profit function is the following

$$
\begin{aligned}
\Pi_{i}(.)= & P_{0} \cdot \sum_{k} q_{i, k}^{0}-\sum_{k} c_{i, k}\left(q_{i, k}^{0}\right)-\sum_{k} F_{i, k}\left(I_{i, k}\right)+u\left[P_{u p}^{1} \cdot \sum_{k} q_{i, u p, k}^{1}-\sum_{k} c_{i, k}\left(q_{i, u p, k}^{1}\right)\right] \\
& +d\left[P_{\text {down }}^{1} \cdot \sum_{k} q_{i, d o w n, k}^{1}-\sum_{k} c_{i, k}\left(q_{i, d o w n, k}^{1}\right)\right]
\end{aligned}
$$

where $P_{0}$ is the initial price and $P_{u p}^{1}$ and $P_{d o w n}^{1}$ are prices in the up/down states respectively in period 1. Then player $i$ 's optimization problem is;

$\max \Pi_{i}\left(q_{i, s}^{t}, I_{i}^{t}, K_{i, s}^{t}\right)$

subject to

$q_{i, s, k}^{t}-K_{i, s, k}^{t} \leq 0$

$$
t=0,1, \forall i, s, k
$$

$Q_{s}^{t}-\sum_{i, k} q_{i, s, k}^{t}=0$

$$
t=0,1, \forall i, s, k
$$

$q_{i, s, k}^{t} \geq 0, K_{i, s, k}^{t} \geq 0$

$t=0,1, \forall i, s, k$

$I_{i, s, k}^{t} \geq 0$

$t=0, \quad \forall i, s, k$

$I_{i, k}^{t}-E\left(I_{i, s, k}^{t}\right)=0$

$t=0, \quad \forall i, s, k$

$K_{i, s, k}^{t+1}-K_{i, s, k}^{t}-I_{i, s, k}^{t}=0$

$t=0, \quad \forall i, s, k$

where $s \in\{u p, d o w n\}$. 
A few remarks regarding the GPS game formulation are in order. The objective function maximizes the profit from the initial period plus expected profits of the future. Note that the equilibrium conditions are imposed for every scenario and every period $t$ because of (4.3). Also, (4.6) enforces the non-anticipativity constraint discussed earlier. After deriving Karush-Kuhn-Tucker (KKT) conditions for the above problem we obtain a linear-complementarity problem, which are the natural form of optimality conditions in inequality-constrained problems. One can employ Argonne National Lab's NEOS serverPATH solver, which is a well known robust and efficient solver for such linear complementarity problems.

We also emphasize that the game formulated in (1), (2), and (3) is exactly equivalent to the GPS game. To see this, note that all strategies that are feasible to the formulation maximizing the sum of discounted payoffs are non-anticipative, and therefore admissible in the GPS model. Moreover, the payoff associated with such strategies is equal in both models. Conversely, all strategies that are feasible to the GPS model are such that the first stage decision of each player can be mapped onto a feasible strategy in the formulation maximizing the sum of discounted payoffs. Furthermore the payoff associated with this strategy is the same, regardless of which model is used for calculating payoffs. Thus there is a one-one map between feasible strategy in each model, and the payoffs are identical. Consequently, these models must provide identical solutions.

\subsection{A Two-Stage Game with Expected Scenarios (GES)}

While there have been some previous attempts at modeling such stochastic equilibria (e.g., Haurie, et al. (1990), Pakes and McGuire (1994)), numerical solution of such games is not as common as their deterministic counterparts. Given this tendency to model games in a deterministic setting, a natural question arises: What differences might one observe in the performance of the players if they all played as though the future were well represented using deterministic models in which expected values were used for decision making? That is, if players replaced the scenario based inverse demand curves with the expected inverse demand curves, then how do they fare in a stochastic environment? We will investigate the consequences of a modeling assumption that 
replaces random variables by their expectation, although the real world may involve uncertainty.

Such issues have already been addressed in the stochastic programming (SP) literature for the case of monopolies. There it is shown that by replacing the probabilistic scenarios by an expected scenario, the monopolist may paint too rosy a picture of future profits, when in fact, the expected profits obtained from such expected value models can be shown to be lower than that obtained from a stochastic decision model (see e.g., Birge and Louveaux (1997)).

We study this issue for oligopolies for the above mentioned game (GES). In the first step investment decisions are made from an expected scenario. In the second step, given these investment decisions, players choose their optimal production decisions. Formally it is defined in the following manner. Let $i$ be a player from a set $N=\{1,2, \ldots, n\}, n$ is finite. In the first step the players view the future in the form of an expected scenario $(\bar{s})$ and make investment decisions as follows.

$$
\bar{I}_{i} \in \arg \max \left\{-F_{i}\left(I_{i}\right)+\left[f_{i}\left(I_{i}, q_{i}^{*}\left(K_{i}^{1}, \bar{s}\right), Q^{*}\left(K_{i}^{1},\left\{K_{j}^{1^{*}}\right\}_{j \neq i} ; \bar{s}\right), \bar{s}\right] \mid I_{i} \in A_{i}\right\}\right.
$$

where

$$
q_{i}^{*}\left(K_{i}^{1^{*}}, \bar{s}\right) \in \arg \max \left\{f_{i}\left(\bar{I}_{i}, q_{i}, Q^{*}\left(K^{1^{*}} ; \bar{s}\right), \bar{s}\right) \mid q_{i} \in B_{i}\left(K_{i}^{1^{*}}\right)\right\},
$$

$Q^{*}\left(K^{1^{*}} ; \bar{s}\right)=\sum_{i=1}^{n} \sum_{k=1}^{m} q_{i, k}^{*}\left(K_{i}^{1^{*}}, \bar{s}\right)$ and,

$f_{i}()=.P\left(Q^{*}\left(K^{1^{*}} ; \bar{s}\right), \bar{s}\right) \sum_{k=1}^{m} q_{i, k}-c_{i}\left(q_{i}\right), \quad K_{i}^{1^{*}}=K_{i}^{0}+\bar{I}_{i}$, and $\bar{s}=E(\tilde{s})$.

In the second step the players choose production quantities for period 0 , and period 1 . In fact, production decisions in period 0 are not affected by investment decisions in period 0 , whereas, productions in period 1 are affected by investments in period 0 . These two decision problems are as follows.

(Period 0): $q_{i}^{*}\left(K_{i}^{0}\right)$ for all $i \in N$ such that when $q_{j} \equiv q_{j}^{*}, j \neq i$ then

$$
q_{i}^{*}\left(K_{i}^{0}\right) \in \arg \max \left\{P\left(Q^{*}\left(K^{0}\right)\right) \sum_{k=1}^{m} q_{i, k}-c_{i}\left(q_{i}\right) \mid q_{i} \in B_{i}\left(K_{i}^{0}\right)\right\}
$$

where $Q^{*}\left(K^{0}\right)=\sum_{i=1}^{n} \sum_{k=1}^{m} q_{i, k}^{*}\left(K_{i}^{0}\right)$ and $B_{i}($.$) is the production set for player i$ and $K_{i}^{0}$ is vector of available capacities from different technologies at time 0 for player $i$.

(Period 1): Production levels are $q_{i}^{*}\left(K_{i}^{1^{*}}, s\right)$ such that

$$
q_{i}^{*}\left(K_{i}^{1^{*}}, s\right) \in \arg \max \left\{f_{i}\left(\bar{I}_{i}, q_{i}, Q^{*}\left(K^{1^{*}} ; s\right), s\right) \mid q_{i} \in B_{i}\left(K_{i}^{1^{*}}\right)\right\},
$$


where $\quad Q^{*}\left(K^{1^{*}} ; s\right)=\sum_{i=1}^{n} \sum_{k=1}^{m} q_{i, s, k}^{*}\left(K_{i}^{1^{*}}, s\right), \quad f_{i}()=.P\left(Q^{*}\left(K^{1^{*}} ; s\right), s\right) \sum_{k=1}^{m} q_{i, s, k}-c_{i}\left(q_{i}\right)$ $K_{i}^{1^{*}}=K_{i}^{0}+\bar{I}_{i}$ and $\bar{I}_{i}$ is determined by the first step of this game.

The information structure of GES is as follows. In period 1 players have the same information in GES as they do in GPS. Each player observes its own capacities and the period 1 demand scenario. In period 0 players in GES believe (or behave as if they believe) that demand will take on a single value (its expected value) in period 1 and that they and other players will choose production in period 1 optimally given that demand level and their capacity. In contrast, in period 0 in GPS players understand that there are two possible demand levels in period 1 and that production in period 1 will be chosen conditional on the demand level.

We are interested in the performance of players under the two alternative games (GPS and GES). Note that even in the presence of uncertainty, the players may play the GES process. Nevertheless, their performance should still be evaluated in a probabilistic setting as explained above. This performance may be expected to be different from that obtained via the GPS process. The question we pose is: Is there a consistent bias? That is, do players playing one of the games always fare better than players involved in the other? Within the SP literature for the case of monopolies, there is a well known result that a model with probabilistic scenarios provides better performance than a model with expected scenarios. The difference between these values is referred to as the "value of the stochastic solution". However an analog of this result in the game setting is unknown.

Suppose that the demand scenarios are modeled by Figure 1. One may interpret $\delta$ in Figure 1 as a volatility level and our characterization suggests that the profitability of the players depends on the game they choose and the volatility of the market. The following lemma applies to both GPS and GES.

Lemma 1: Consider a market with $n$ firms facing a future that is described by two states: an "up" state, and a "down" state. In this market, we assume that the inverse demand curve is linear in either state, and these curves have the same slope, regardless of the state. Moreover, suppose that cost function of each firm, denoted $c_{i}$, can be expressed as the sum of convex separable functions; that is, $c_{i}\left(\sum_{k} q_{i, k}\right)=\sum_{k} g_{i, k}\left(q_{i, k}\right)$, where $g_{i, k}$ are 
convex and differentiable. Then under either the GPS game or the GES game, each player produces at least as much quantity in the "up" as than in the "down" state.

Proof: Let $q_{i, k, u}$ and $q_{i, k, d}$ denote the quantities produced by firm $i$ using technology $k$ in the "up" and "down" states respectively. The claim is that $\sum_{k} q_{i, k, u} \geq \sum_{k} q_{i, k, d}$ for all $i$. Contrary to the claim, suppose that there exists an index $I$ for which $\sum_{k} q_{I, k, u}<\sum_{k} q_{I, k, d}$. Then, there must exist a technology index $K$ such that $q_{I, K, u}<q_{I, K, d}$. Now the KKT conditions for each firm imply that $P\left(Q_{s}\right)+q_{I, K, s} P^{\prime}\left(Q_{s}\right)-g_{I, K}^{\prime}\left(q_{I, K, s}\right)-\mu_{I, K, s}=0, \quad s=u, d$ where $Q_{s}=\sum_{i, k} q_{i, k, s}$. Since $q_{I, K, u}<q_{I, K, d}$, it follows that $\mu_{I, K, u}=0$. It follows that

$$
P\left(Q_{u}\right)+q_{I, K, u} P^{\prime}\left(Q_{u}\right)-g_{I, K}^{\prime}\left(q_{I, K, u}\right)=0 .
$$

On the other hand, $\mu_{I, K, d} \geq 0$ implies that

$$
P\left(Q_{d}\right)+q_{I, K, d} P^{\prime}\left(Q_{d}\right)-g_{I, K}^{\prime}\left(q_{I, K, d}\right) \geq 0 .
$$

Using the KKT conditions for the pair $I, K$, the supposition that $q_{I, K, u}<q_{I, K, d}$, and $P^{\prime}\left(Q_{u}\right)=P^{\prime}\left(Q_{d}\right)<0$, we have $P\left(Q_{u}\right)=-q_{I, K, u} P^{\prime}\left(Q_{u}\right)+g_{I, K}^{\prime}\left(q_{I, K, u}\right)<-q_{I, K, d} P^{\prime}\left(Q_{d}\right)+g_{I, K}^{\prime}\left(q_{I, K, d}\right) \leq P\left(Q_{d}\right)$.

Due to the monotonicity of the (linear) inverse demand curve, we conclude that under our supposition, $Q_{u}>Q_{d}$. But this inequality implies the existence of another pair, say $J, T$ such that $q_{J, T, d}<q_{J, T, u}$. Using the same arguments as above, we then come to the conclusion that $Q_{d}>Q_{u}$. These contradictory conclusions imply that there cannot exist any index $I$ for which $\sum_{k} q_{I, k, u}<\sum_{k} q_{I, k, d}$, and hence the result.

The following proposition also holds for both games.

Proposition 1: Assume that the probability of the "up" state is at least as high as the probability of the "down" state and consider a case in which all players experience identical quadratic costs and capacities. Moreover, assume that equilibrium involves unconstrained production in the low-demand state. Then players' equilibrium payoffs are increasing as demand volatility increases. 
Proof: This proof is for the case in which each firm has a single technology. The proof for the case of multiple technologies is similar. Given $P_{0}=D-Q_{0}, P_{u}^{1}=D+\delta-Q_{u}^{1}$, $P_{d}^{1}=D-\delta-Q_{d}^{1}$, the Lagrangian function for player $\mathrm{i}$ is;

$$
\begin{aligned}
L_{i}= & \left(D-\sum_{i} q_{i}^{0}\right) q_{i}^{0}-c\left(q_{i}^{0}\right)-F\left(I_{i}^{0}\right)+u\left[\left(D+\delta-\sum_{i} q_{i, u}\right) q_{i, u}-c\left(q_{i, u}\right)\right]+d\left[\left(D-\delta-\sum_{i} q_{i, d}\right) q_{i, d}-c\left(q_{i, d}\right)\right] \\
& +\lambda_{i, 0}\left(K_{i}^{0}-q_{i}^{0}\right)+\lambda_{i, 1}\left(K_{i}^{0}+I_{i}^{0}-q_{i, u}\right)+\lambda_{i, 2}\left(K_{i}^{0}+I_{i}^{0}-q_{i, d}\right) .
\end{aligned}
$$

The total derivative of above expression with respect to $\delta$ is;

$\frac{d L_{i}}{d \delta}=\frac{\partial L_{i}}{\partial q_{i}^{0}} \frac{\partial q_{i}^{0}}{\partial \delta}+\frac{\partial L_{i}}{\partial q_{i, d}} \frac{\partial q_{i, d}}{\partial \delta}+\frac{\partial L_{i}}{\partial q_{i, u}} \frac{\partial q_{i, u}}{\partial \delta}+\frac{\partial L_{i}}{\partial I_{i}^{0}} \frac{\partial I_{i}^{0}}{\partial \delta}+\frac{\partial L_{i}}{\partial \delta}+\sum_{j \neq i} \frac{\partial L_{i}}{\partial q_{j, d}} \frac{\partial q_{j, d}}{\partial \delta}+\frac{\partial L_{i}}{\partial q_{j, u}} \frac{\partial q_{j, u}}{\partial \delta}+\frac{\partial L_{i}}{\partial q_{j}^{0}} \frac{\partial q_{j}^{0}}{\partial \delta}$

$=0+0+0+0+u \cdot q_{i, u}\left[1-\sum_{j \neq i} \partial q_{j, u} / \partial \delta\right]-d \cdot q_{i, d}\left[1+\sum_{j \neq i} \partial q_{j, d} / \partial \delta\right] \quad$ (by first order necessary

conditions),

Case 1.Up-state production is unconstrained.

Let $c^{\prime}(q)=a+b q, F^{\prime}(I)=g+h I$, with non-negative coefficients $a, b, g, h$. From the first order necessary conditions

$D-2 q_{i}^{0}-a-b q_{i}^{0}-\sum_{j \neq i} q_{j}^{0}=0$. Hence $q_{i}^{0^{*}}=(D-a) /(n+1+b) . \quad$ Similarly,

$q_{i, u}^{*}=(D+\delta-a) /(n+1+b), q_{i, d}^{*}=(D-\delta-a) /(n+1+b)$. Thus $\partial q_{i, u}^{*} / \partial \delta=1 /(n+1+b)$,

$\partial q_{i, d}^{*} / \partial \delta=-1 /(n+1+b)$, and by the result of Lemma 1 and $u \geq d$, it is easy to see that $d \Pi_{i} / d \delta=d L_{i} / d \delta>0$.

Case 2. Up-state production is constrained.

Then $q_{i, u}^{*}=K_{i}^{0}+I_{i}^{0^{*}}$, and ${F^{\prime *}}^{*}()=.g+h\left(q_{i, u}^{*}-K_{i}^{0}\right)$, and the first order condition becomes $u\left[D+\delta-2 q_{i, u}-a-b q_{i, u}-\sum_{j \neq i} q_{j, u}\right]-g-h\left(q_{i, u}-K_{i}^{0}\right)=0$, which implies

$q_{i, u}^{*}=\left[u(D+\delta-a)-g+h K_{i}^{0}\right] /[u(n+1+b)+h]$. Thus $\partial q_{i, u}^{*} / \partial \delta=1 /(n+1+b+h / u)$ and

$d L_{i} / d \delta=u q_{i, u}^{*}[1-1 /(n+1+b+h / u)]-d q_{i, d}^{*}[1-1 /(n+1+b)]>0$.

Example1: The aim of this example is to compare GPS and GES games in terms of their performance as volatility changes. Suppose that two technologies are available for each player. Specifically, initially each player has 3 units of the technology 1 capacity, (i.e. $K_{i, 1}=3$ ), and has 2 units of technology 2 capacity, (i.e. $K_{i, 2}=2$ ). Investment and 
production $\quad$ cost $\quad$ functions $\quad$ are $\quad F_{i, 1}()=.C_{i, 1} I_{i, 1}$ and $c_{i, 1}()=.C_{i, 1} q_{i, 1}^{2}, \quad$ and $F_{i, 2}()=.C_{i, 2} I_{i, 2}^{2}$ and $c_{i, 2}()=.C_{i, 2} q_{i, 2}, C_{i, m}=i+1$, for technologies 1 and 2 respectively, and $i=1,2,3,4,5$. Each player $i$ predicts a market with the scenarios in Figure 1, where $D=90$, $u=0.5$ is the probability of the "up" state, and $d=0.5$ is the probability of the "down" state. We investigate cases when $\delta$ assumes the following values: 0,5,10,15,20,25,30. In GES, in the first step each player solves (5) with $\bar{s}=E(\tilde{s})$, in that case $\mathrm{s}=1$. From here each player implements this investment decision to the following second step optimization problem.

$\max \Pi_{i}\left(q_{i, s}^{t}, \bar{I}_{i}^{t}, K_{i, s}^{t}\right)$

subject to

$q_{i, s, k}^{t}-K_{i, s, k}^{t} \leq 0 \quad t=0,1, \forall i, s, k$

$Q_{s}^{t}-\sum_{i, k} q_{i, s, k}^{t}=0 \quad t=0,1, \forall i, s, k$

$q_{i, s, k}^{t} \geq 0, K_{i, s, k}^{t} \geq 0 \quad t=0,1, \forall i, s, k$

$\bar{I}_{i, s, k}^{t} \geq 0 \quad t=0, \quad \forall i, s, k$

$K_{i, s, k}^{t+1}-K_{i, s, k}^{t}-\bar{I}_{i, s, k}^{t}=0 \quad t=0, \quad \forall i, s, k$

In Figure 2, we illustrate player 3's expected profit values with respect to volatility changes under both games. Because all players have similar cost functions, we observe similar figures for other players too. Note that for each volatility level, GES dominates GPS. In GPS, there is an investment opportunity as demand volatility increases. In contrast, greater demand volatility has no impact on investment decisions in GES since the expected level of demand is unchanged. As demand volatility increases, players in the GPS game increase their investments in order to take advantage of higher demand in the good demand state; they can leave extra capacity unutilized in the low demand state. We also note that changing $\delta$ has no effect at all on ex ante (that is the first step of GES) expected profit. That is, the expected profit at the time the investment decision is being taken does not change in $\delta$. On the other hand, if we take the average of profits after period one demand realizations and after firms make their period one production adjustments, then profit is impacted by change in $\delta$. That is, ex post average profit for the GES game is increasing in $\delta$. 


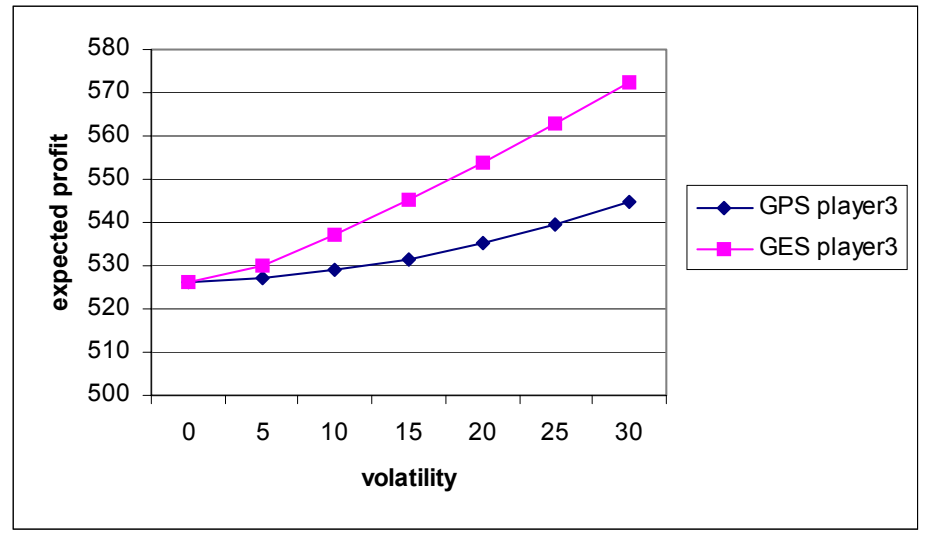

Figure 2: Player 3's performance under these games

\subsection{A two-stage Hybrid Game (HG)}

In this subsection, we show that despite the possible advantage of GES over GPS, the former is unstable because it creates a situation in which one of the players may have an incentive to deviate. In order to see this, we consider a hybrid game (HG) which is a combination of GPS and GES in the following manner.

In the first step of the hybrid game all players assume that the others play according to the first step of GES. However, player $i$ chooses to deviate by planning for investments by solving a decision model in which the probabilistic scenarios are included. Once the investments are in place, the second step of the game proceeds as before. It is not difficult to see that as long as GPS and GES have solutions, the hybrid game also has solutions. In such a hybrid game, we can show that player $i$ will always be better off.

Proposition 2 : If a player plays HG (i.e. a player who deviates from GES and plays GPS and others play GES) then this player's expected profit function is ordered as $\Pi_{G E S} \leq \Pi_{H G}$ under the assumptions made above regarding demand and technology conditions.

We will give a sketch of the proof. Since all non-deviating players have committed their decisions then the game reduces to a single player stochastic program. Applying a basic result (proposition 1 of Birge and Louveaux (1997), page 140) from stochastic programming it follows that the expected profit of the deviating player in the 
stochastic program exceeds that from the expected value model. The above result and the corresponding arguments hold in the multi-stage game (see section 3).

With the insight gained from the $\mathrm{HG}$, let us reconsider the conclusions from Example 1. If only one player reacted to the positive volatility, while the other players retained their GES investment levels, then this player would increase their investment and their expected profit would rise. This increase in expected profit corresponds to the value of the stochastic solution, referred to earlier. But in a multi-player game setting, players' payoffs are interdependent. In fact all of the players will increase their investments and their outputs. The net effect is that prices and expected profits are lower in the GPS game than in the GES game. This can be viewed as a prisoners' dilemma situation. Players in the GPS game end up being worse off by pursuing what is individually optimal (given strategies of rival players) compared to players in the GES game who behave sub-optimally from an individual point of view, but more nearly optimally from the point of view of collective profits.

\section{Multi-stage Stochastic Programming Games}

In this section we study multi-stage games that are generalizations of the two stage games presented in section 2. Multi-stage games call for sequential decisionmaking on the part of each player, and the calculation of dynamic equilibrium is a nontrivial undertaking. The difficulties are compounded when there are lags in the system dynamics, which is common in situations where investments in capacity are followed by several periods of construction prior to production. In this section, we will show that the SP framework introduced in the previous section (for two-stage models) can be extended to the multi-stage case without significantly adding to the computational burden of calculating S-adapted open-loop equilibrium. The key observation that makes this extension possible is the introduction of multi-stage non-anticipativity constraints, which happen to be linear, and are easily incorporated in numerical methods. Thus the SP approach opens up the possibility of computing S-adapted open-loop equilibrium for much larger problems than is possible using dynamic programming (DP). We demonstrate the applicability of this methodology through a study which predicts equilibrium electricity prices for the Ontario whole-sale electricity market. To the best of 
our knowledge, such studies of dynamic equilibrium, using realistic market data, have not been possible because of the exclusive reliance on DP. However, the SP approach of this paper provides an effective methodology for predicting dynamic equilibrium. In section 3.4, we will also provide some comparisons between standard DP, and the methodology of this paper.

\subsection{A Model of Demand in the Multi-stage Case}

The scenario tree used in section 2 is highly simplified, because there was only one future stage with two possible outcomes. In the multi-stage formulation, the scenario tree can become considerably more complex. In Figure 3, we give an example of scenario tree, which captures the sequence in which information is revealed. Also, in the class of models we are considering (i.e., Stochastic Programming) decisions are not allowed to alter the underlying stochastic process. So the underlying stochastic process is assumed to be exogenous to the decision process.

In multi-stage formulations the demand function may be modeled as follows. Suppose that demand follows $N\left(\mu, h^{2}\right)$ within each time period. To approximate this demand distribution in the scenario tree, we use the properties of $m$-point $\left(m=2^{t}\right)$ discrete uniform distribution. The price-quantity relationship is assumed to be governed by the form $a P_{t}+b Q_{t}=D+\tilde{\delta}_{t}, \quad$ and for $t=0, \quad \tilde{\delta}_{0}=0$ and for $t=1,2,3, \ldots, \mathrm{T}$, $\tilde{\delta}_{t}=\left\{\mp(2 w-1) g_{t}\right\}_{w=1}^{2^{t-1}}, \quad$ where $\quad a, b, D>0$ are given constants, and $g_{t}=h / \sqrt{2^{1-t} \sum_{w=1}^{2^{t-1}}(2 w-1)^{2}} . P, Q \in \mathfrak{R}_{+}$and denote the market price and total output, respectively, and $\tilde{\delta}$ is the random variable. Figure 3 illustrates the behavior of a demand function. 


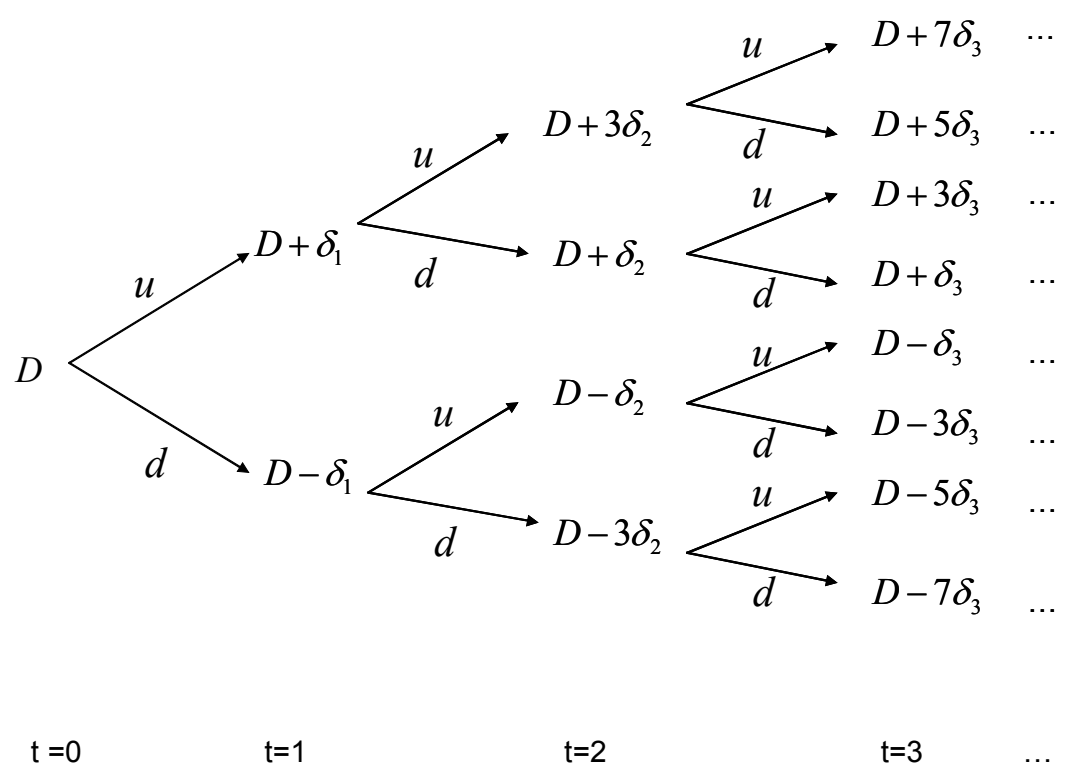

Figure 3: Scenario-tree representation of demand function

Conditional probabilities are given on the arcs, with the upside probability denoted as ' $u$ ', and the downside probability denoted as ' $d$ ' and of course $u+d=1$. In Section 3.3., to predict Ontario wholesale electric prices we will use this demand structure (given that $u=1 / 2$ ).

\subsection{A Multi-Stage Game with Probabilistic Scenarios}

Multi-stage games are multi-period extension of two-stage games. At any given period $t, t$ is finite, players simultaneously choose investment and production decisions from multiple technologies. As in the previous section, investment in one period will only become available as productive capacity in the next period. There are more general lags that can also be modeled within this framework. However we omit such generalizations in the interest of brevity.

The setting for our formulation is one in which each player can only observe production output of the other players through the market price. We refer the reader to Haurie, et al. (1990) for a recursive formulation. On the other hand the non-recursive formulation is as follows. Let $M(o)$ represent scenarios passing through node $o$ of the scenario tree. As before let $I_{i, s}^{t}, q_{i, s}^{t} \in \mathfrak{R}_{+}^{m}$ denote investment and production quantities associated with $m$ technologies. The total expected profit for a player is denoted 
$\Pi_{i}=\sum_{s} p_{s} \Pi_{i, s}$, where $p_{s}$ is the probability of each scenario. Formally we define this game as follows.

For a given scenario $s \in S=\{1,2,3, \ldots, \omega\}$, each player $i$ solves following problem: $\underset{\substack{q_{i, s}^{t}, I_{i, s}^{t} \\ q_{t}}}{ } \sum_{t} \Pi_{i, s}^{t}\left(q_{i, s}^{t}, I_{i, s}^{t}, K_{i, s}^{t}, P_{s}^{t}\right), t=0,1,2,3, \ldots, \mathrm{T}$,

subject to $K_{i, s}^{t+1}=K_{i, s}^{t}+I_{i, s}^{t}$,

and subject to $E\left(I_{i, s}^{t} \mid M(o)\right)=I_{i}^{t}, q_{i, s}^{t} \leq K_{i, s}^{t}$,

where in (8) we have used a standard one period lag. However if there are more complicated time lags, then (8) needs to be modified to suit the situation being modeled; nevertheless the remainder of the model as well as associated algorithms remains unchanged.

Other applications in which this formulation may be advantageous include situations in which a player's objective is not separable by time period. For instance, if the players' objectives include risk preferences then such models may not be separable, and once again a non-recursive formulation becomes necessary.

A solution algorithm for the non-recursive formulation is as follows:

i) The Karush- Kuhn-Tucker conditions for the problem are as follows: $\forall i, t, s$

$L_{i, s}=\sum_{t}^{T} \beta(t)\left\{P_{s}^{t}\left(Q_{s}^{t}\right) q_{i, s}^{t}-c_{i}\left(q_{i, s}^{t}\right)-F_{i}\left(I_{i, s}^{t}\right)+\alpha_{i, s}^{t}\left[K_{i, s}^{t}-q_{i, s}^{t}\right]+\lambda_{i, s}^{t}\left[E\left(I_{i, s}^{t}\right)-I_{i}^{t}\right]\right\}$

$q_{i, s}^{t} \frac{\partial L_{i, s}}{\partial q_{i, s}^{t}}=0, I_{i, s}^{t} \frac{\partial L_{i, s}}{\partial I_{i, s}^{t}}=0, \alpha_{i, s}^{t} \frac{\partial L_{i, s}}{\partial \alpha_{i, s}^{t}}=0$

$q_{i, s}^{t} \leq K_{i, s}^{t}, \quad I_{i, s}^{t}-E\left(I_{i}^{t}\right)=0$

$K_{i, s}^{t}=K_{i, s}^{t-1}+I_{i, s}^{t-1}, \quad Q_{s}^{t}-\sum q_{i, s}^{t}=0$

$K_{i}^{0}=\kappa \geq 0, q_{i, s}^{t} \geq 0, I_{i, s}^{t} \geq 0$,

where $\left(\alpha_{i, s}^{t} \geq 0\right),\left(\lambda_{i, s}^{t} \geq 0\right)$ denote for dual prices of inequalities and non-anticipative conditions respectively, and $\beta(t)$ is a discount factor. ${ }^{2}$

ii) Use the PATH solver for a complementarity problem.

\footnotetext{
2 In example 1 we assume the discount factor to be 1 , since the problems involve a fairly small number of stages.
} 
Proposition 3: For the case of a one-period lag between investment and production, the recursive formulation is equivalent to the non-recursive formulation for oligopolistic games.

Proof: The recursive formulation for the multi-period GPS game (hereafter NF) for a firm $i$ may also be defined in the following manner:

$$
\begin{aligned}
& \max \sum_{o} p_{o} \Pi_{o}^{i}\left(x_{o}^{i}\right) \\
& \text { s.t. } \sum_{m \in A_{o}} I_{o, m}^{i}+K_{0}^{i} \geq q_{o}^{i} \quad, \quad \forall o \\
& q_{o}^{i}, x_{o}^{i} \geq 0, K_{0}^{i}>0
\end{aligned}
$$

where, $x_{o}^{i}=\left(I_{o}^{i}, q_{o}^{i}\right), \Pi_{o}^{i}()=.\mathrm{P}_{o} q_{o}^{i}-c\left(q_{o}^{i}\right)-F\left(I_{o}^{i}\right)$, and $A_{o}$ denotes for ancestors of node $o$. The non-recursive formulation of the multi-stage GPS game (hereafter SF) for a firm $i$ may also be defined as follows:

$$
\begin{array}{ll}
\max & \sum_{s} p_{s} \sum_{t} \Pi_{t s}^{i}\left(x_{t s}^{i}\right) \\
\text { s.t. } & \sum_{m \in A_{s t}} I_{o, m}^{i}+K_{0}^{i} \geq q_{t s}^{i}, \quad \forall t, s \\
& x_{t s}^{i}=E\left(\tilde{x}_{t}^{i}\right) \\
& q_{t s}^{i}, x_{t s}^{i} \geq 0, K_{0}^{i}>0
\end{array}
$$

where, $x_{t s}^{i}=\left(I_{t s}^{i}, q_{t s}^{i}\right), \Pi_{t s}^{i}()=.\mathrm{P}_{t s} q_{t s}^{i}-c\left(q_{t s}^{i}\right)-F\left(I_{t s}^{i}\right)$

Let $x_{o}^{i}$ be a solution for NF and let $M_{o}$ be the scenarios that pass through the node $o$, then for $t_{o}=t$ define the following relation; $x_{o}^{i}=x_{t_{o} s}^{i}$ for all $s \in M_{o}$. That means $I_{t_{o} s}^{i}=I_{o}^{i}$ and $q_{t_{o} s}^{i}=q_{o}^{i}$ by definition of $x_{o}^{i}$. But these two equalities are just nonanticipativity constraints of SF, and so that $\sum_{t} \Pi_{t s}^{i}()=.\Pi_{o}^{i}($.$) , then we see that proposed$ solution is a feasible solution of SF. By existence and uniqueness theorems this feasible solution is same as the solution of NF. The reverse is similar. Because, if we have nonanticipative solutions, then the fact that the $I, q$ are non-anticipative imply that they are constant at any node, thus letting us set the value of the node variable for $I, q$ to assume the same value as that of all scenarios passing through node $o$. 
Proposition 4: The non-cooperative games defined in this paper together with the above demand and technology conditions admit a unique solution.

For a proof see Haurie, et al. (1990) or see the proofs of theorems 7.1 and 7.7 in Friedman (1977).

\subsection{Application to the Ontario wholesale electricity market}

In November 1998, the Government of Ontario passed the Energy Competition Act, which divided Ontario Hydro into several companies including the Independent Electricity Market Operator (IMO), Hydro One, and Ontario Power Generation (OPG). The Act aimed at creating competitive market, and after extensive debates the wholesale Ontario electricity market commenced on May 1,2002. Currently the IMO is the market administrator and responsible for a fair and competitive marketplace. In the process of making sure that supply matches demand, the IMO is also responsible for reliability issues. Hydro One mainly transmits power, while OPG generates most of the electricity consumed. Prior to the opening of the wholesale market, OPG divested some nuclear plants and gas-fueled plants to independent generation companies. The Ontario Energy Board (OEB) regulates the rates that end-user customers pay. In Table 1 we present Ontario's existing generation resources, installed capacities, ownership and percentage of the capacities (see www.theimo.com).

Table 1: Ontario's existing power generation structure

\begin{tabular}{|c|c|c|c|}
\hline Ownership & Technology & Installed Capacity, MW & $\%$ of total \\
\hline Bruce & nuclear & 4,728 & 15.5 \\
NUGA & oil/gas & 2,224 & 7.3 \\
OPG & nuclear & 6,103 & 20 \\
OPG & green & 66 & 0.2 \\
OPG \& affiliates & hydroelectric & 7,676 & 25.2 \\
OPG & oil/gas & 2,140 & 7 \\
OPG & coal & 7,564 & 24.8 \\
\hline
\end{tabular}

In this table, we have designated wind, solar, biomass and geothermal generation resources as "green" technology. The total installed generation capacity in Ontario 
amounts to 30,501 MW (megawatt energy). OPG owns three (comprising of four units at Darlington, four units at Pickering B and one unit at Pickering A) out of five nuclear stations. Other nuclear generation facilities owned by Bruce Nuclear (hereafter Bruce), which is comprised of Bruce A and Bruce B, is Ontario's the largest independent power generator. All hydroelectric generation in Ontario belongs to OPG - Niagara, Northeast, Northwest, Ottowa/St.Lawrence groups and OPG Evergreen Energy Division. All of the five coal fired stations (Atikokan, Lakeview, Lambton, Nanticoke, and Thunder Bay) are also owned by OPG. OPG also owns Lennox Generating Station, which is fueled by natural gas and oil. The other less significant power source is wind power. OPG owns three wind stations, and its capacity is estimated to be less than $50 \mathrm{MW}$. There are other non-utility generators (NUG) in Ontario that are mostly fueled by natural gas and oil. According to APPrO (Association of Power Producers of Ontario), the NUG have 1307 MW capacities of gas fueled generation stations. Also ATCO Power Ltd., the giant Alberta utility has recently been operating a $625 \mathrm{MW}$ plant in Windsor, Ontario. This plant is a natural gas fired plant. In addition, Imperial Oil Ltd and Northland Power Inc installed a total of $130 \mathrm{MW}$ gas-fired turbines in 2004 (in Ontario). All the capacity data reported above are obtained from IMO, OPG, APPrO, Hydro-One web pages. IMO states that the rest of the suppliers are small and some are already affiliated with OPG and some are independent generators. In Table 1, NUGA refers to the total power provided by NUG, ATCO Power, Imperial Oil and Northland Power Inc.

Using the SP modeling paradigm, we perform an analysis to forecast capital investment decisions. We assume that each time stage, $t$, represents a year and run the model for five years. Note that we do not model the impact of forward contracts on the spot market since there is currently no forward market in Ontario. We obtained hourly demand data from May 1, 2002 (opening of the competitive wholesale market) to May 1, 2004 and transformed it to average hourly value for the day and hourly value for the year. In the analysis time stage $t=0$ corresponds to May 1, 2004 - May 1, 2005, similarly other stages. We focus on suppliers OPG, Bruce, NUGA and treat NUGA as a single supplier. Energy supply distribution (terawatt hour) by fuel type between May 2003 and April 2004 were $42 \%$ nuclear, $23 \%$ hydro, $21 \%$ coal, $8 \%$ other (including natural gas, oil, wind, solar, biomass) and 6\% imports (see IMO year in review report 2003-2004). 
According to National Energy Board's energy market assessment report, Market Power Mitigation Agreement (MPMA) was developed to reduce OPG's share of the generation market. It is required to reduce its fossil generation capacity and to have at most $35 \%$ of the total generation capacity.

We consider two cases: one with three players in the market, the other with five players in the market. In Ontario, a five-dominant-player market is expected as a result of divestiture of the largest supplier OPG.

In Table 2, we represent the players, their generation technologies, the upper bounds of available in-use capacities, and average variable operation and maintenance costs of the technologies. In the second case, the five-player market, we will assume that OPG will keep its hydro, nuclear and green power facilities.

Table 2: The Ontario market player-technology-cost-capacity structure.

\begin{tabular}{|l|l|c|c|}
\hline Suppliers & Technology & Max.in_use_cap (MWe) & Cost (\$/MWh) \\
\hline Bruce & nuclear & 3,329 & 4.56 \\
NUGA & oil/gas & 1,458 & 25.46 \\
& nuclear & 4,298 & 4.56 \\
\multirow{3}{*}{ OPG } & green & 66 & 0 \\
& hydro & 4,177 & 0 \\
& oil/gas & 1,431 & 25.46 \\
& coal & 3,814 & 21.96 \\
\hline
\end{tabular}

Based on the above energy supply distribution we calculated available in usecapacities of each player. Since in general marginal units that cleared the market are gas and oil fueled generators, and OPG and NUGA have oil/gas plants, we attributed imports (from the interconnecting grid of other jurisdictions such as New York, Michigan, Minnesota, and Manitoba) to them, and increased their maximum in use capacities for oil/gas plants at the average level of imports.

Table 2 implies that almost $70 \%$ of the installed nuclear generation, $55.4 \%$ of the total hydroelectric capacity, $50.4 \%$ of the total installed coal turbine generation capacity, $31.8 \%$ capacity of oil/gas fired stations are available on average to meet the demand for each hour. We also assume that green technology works with $100 \%$ capacity all the time. 
In the last column of Table 2 we present the cost structures of the technologies. Firm level cost data in general is private and not disclosed. Thus we could not obtain all detailed information such as type and brand names of the generators, their heat rates per year, heat rates $n^{\text {th }}$ of-a-kind, overnight costs, fixed operation and maintenance costs, and contingency factors. However we obtained variable operation and maintenance costs of the technologies. This information is obtained from Energy Information Administrationassumptions to the annual energy outlook 2004 booklet. All prices are inflation adjusted (we assumed 3\% inflation per year) and are in year 2004 dollars. Above oil/gas price represents average price of IGCC (integrated gasification gas combined cycles) with carbon sequestration, convertible gas/oil combined cycle, and advanced combined cycle with carbon sequestration type technologies. We assume constant marginal cost of production up to capacity.

For capital investment decisions, we use the following overnight construction costs of the technologies provided by Taylor (2002) (These costs are very close to the cost figures defined by Energy Information Administration-assumptions to the annual energy outlook 2004 booklet). These costs include contingency factors, technological optimism and learning factors, but exclude regional multipliers.

\section{Table 3: Overnight construction costs by technology (\$/KWe)}

\begin{tabular}{|c|c|}
\hline Technology & 2005 year startup costs \\
\hline nuclear & 1600 \\
coal & 1080 \\
large hydro & 1800 \\
gas/oil & 450 \\
green(wind) & 800 \\
\hline
\end{tabular}

We assume constant marginal cost of adding capacity. These costs are presented in terms of US\$; we assumed same costs in CA\$ because of similar purchasing power per consumption basket.

We use a linear demand with constant slope and varying intercepts similar to Green and Newbery (1992), Wolfram (1999), and Garcia-Diaz and Marin (2003), 
however we use scenario tree representation of a demand model that depicted in Figure 3, in which we assume equal 'up' and 'down' state probabilities.

For short run analysis of prices, random walk models may not explain the behavior of the energy prices. Instead, mean reverting processes are recommended because it is assumed that the market price might converge to its marginal cost price after several jumps. In order to distinguish between short term and long term price processes, we used Dickey-Fuller unit root tests on daily, weekly, monthly and quarterly wholesale electric prices in the Ontario market from May 1, 2002 to September 14, 2004. We observe that as time interval enlarges and sample size decreases prices tend to follow random walk. (This observation is consistent with the literature, e.g., see Dixit and Pindyk (1997)). Specifically we find that, mean reverting process captured only for daily and weekly prices. However, with the random walk approach, as time period increases the volatility of prices tend to explode, which is the main criticism of this approach to predict energy prices. Hence, we employ $m$-point discrete uniform distribution to approximate a Normal distribution given that hourly MW for the year is normally distributed. Specifically we utilize the following.

Let the hourly load for a year be distributed $N\left(\mu, h^{2}\right)$. We calculate $h=1,089 \mathrm{MW}$ based on the IMO predictions on the load volatility for the years 2005 through 2014. The average hourly price for the year (period) May 1, 2003-Apr 31, 2004 is $\$ 48.2$, and hourly load for the year is $18,055 \mathrm{MW}$. Assume that $Q_{t}(p)=\alpha_{t}-\beta p$ denotes electricity demand, in which $Q_{t}(p)$ is the hourly load for time (year) $t$ at the price $p$. Also assume that price elasticity of demand be 0.2 . This elasticity levels is commonly assumed for the wholesale electricity markets (see, for example, Wolfram (1999), Taylor (1975), Branch (1993)). Then we calculate $\beta=75$. Let $\alpha_{t}=D\left(1+\rho_{e}\right)^{t}+\delta_{t}$, where $\left(\rho_{e}\right)_{e}=\{0.006,0.009,0.013\},(e=$ low, medium, high $)$, are the growth rates of the demand that IMO expects for the future per year. Given the above information, for $t=0$ (initial node), in which $\delta_{0}=0$, we calculate that $D=21,670$. Hence in the initial node we have the demand function $Q_{0}(p)=21,670-75 p$. Accordingly, the $m$-point discrete uniform distribution described in Section 3.1 will use 
an "up" demand scenario of $Q_{1, u p}(p)=D\left(1+\rho_{e}\right)+h-\beta p$ for $t=1$, and a "down" demand scenario will be $Q_{1, \text { down }}(p)=D\left(1+\rho_{e}\right)-h-\beta p$, with equal probabilities $1 / 2$ and $1 / 2$. Demand scenarios for other periods are obtained by the process that is described in Section 3.1.

Each player's optimization problem is similar to the formulation of (4.1) - (4.7). (For the sake of brevity we do not rewrite the optimization problem for each player.) We extend the time to five stages $(t=0,1,2,3,4)$ and will allow depreciation in technologies for each period and add salvage value to the profit at the last period. To implement this, we assume that each technology has 30-year life period and each technology depreciates linearly in each year. Formally, we replace capacity constraint (4.7) with $K_{i, s, k}^{t+1}=K_{i, s, k}^{t}(1-r)+I_{i, s, k}^{t}$ for all $t, i, s, k$, where in general $r \in\{0,1 / 30\}$. A salvage value for each type of technology equal to a high percentage of the replacement of the capacity (e.g., $90 \%$ of the replacement cost) is added to the profit function of each player $i$ at time $t=4$. That is, we will add $\sum_{s, k} p_{s} K_{i, s, k}^{t} F_{i, s, k}^{\prime} v$ to the profit, where $v=0.9$, $p_{s}$ is a probability of scenario $s, F^{\prime}$ is the installment (or replacement) cost of each unit of technology.

We model the market as a game with probabilistic scenarios and compute the unique S-adapted open-loop equilibrium. Admittedly, in this simplified version of Ontario wholesale electricity market model we assumed that all generators run continuously during the study period, however in reality this holds only for nucleargenerated power. Hydroelectric generators may provide base-load and peak generation depending on the level of water availability. Coal generators start up early morning and run till late in a day and natural gas and oil plants usually used to meet the peak demand.

Example 2: We use the setup in Tables 2 and 3 and run the stochastic programming model using PATH solver. We make two sets of runs. In each run we compare the equilibrium results of two-cases; the three-player market versus the five-player market, for each level of growth in market demand (low-growth, medium-growth, high-growth in demand). The five-player case involves the case in which OPG divests its oil/gas generation facilities to Player 4 and its coal fired units to Player 5 in the beginning of the 
game. (For those players' capacity and cost levels see the last two rows of the Table 2.) Again first three players are OPG, Bruce and NUGA. This divestiture is a likely scenario to be expected in the market, and is consistent with the Market Power Mitigation Agreement in Ontario.

In Tables $4 \mathrm{a}$ and $4 \mathrm{~b}$ we report the first run (no capacity depreciation - no salvage value case) equilibrium price outcomes, expected price, standard deviation, maximum and minimum prices ( $\$ / \mathrm{MWh}$ for each year). We observe that when number of players increased from three to five, yearly average market price reduced $28 \%$ and yearly average price volatility decreased $23 \%$. Since demand growth levels are small and close to each other, equilibrium prices are similar to each other across demand growths. The difference between maximum and minimum price levels is also small.

Table 4a. Yearly price levels with 3 players (no salvage value $\&$ no depreciation)

\begin{tabular}{|c|c|c|c|}
\hline \multicolumn{4}{|c|}{ Demand Growth } \\
\hline Period & Low $(0.6 \%)$ & Medium $(0.9 \%)$ & High $(1.3 \%)$ \\
\hline $0=4.1 .04-05$ & $(114.8,-, 114.8,114.8)$ & $(114.8,-, 114.8,114.8)$ & $(114.8,-, 114.8,114.8)$ \\
$1=4.1 .05-06$ & $(95.8,10.2,103.1,88.5)$ & $(95.7,10.2,103.0,85.5)$ & $(95.7,10.3,102.9,88.4)$ \\
$2=4.1 .06-07$ & $(96.0,7.6,105.0,87.0)$ & $(96.3,7.6,105.3,87.3)$ & $(96.7,7.5,105.6,87.8)$ \\
$3=4.1 .07-08$ & $(96.9,7.1,107.3,86.5)$ & $(97.6,7.1,108.0,87.3)$ & $(98.7,7.0,108.9,88.4)$ \\
$4=4.1 .08-09$ & $(97.8,6.9,108.9,86.6)$ & $(99.0,6.8,110.0,87.9)$ & $(100.6,6.7,111.6,89.6)$ \\
\hline \multicolumn{4}{|c}{} \\
\hline
\end{tabular}

Table 4b. Yearly price levels with 5 players (no salvage value \& no depreciation)

\begin{tabular}{|c|c|c|c|}
\hline \multicolumn{3}{|c|}{ Demand Growth } \\
\hline Period & Low $(0.6 \%)$ & Medium $(0.9 \%)$ & High $(1.3 \%)$ \\
\hline $0=4.1 .04-05$ & $(79.8,-, 79.8,79.8)$ & $(79.8,-, 79.8,79.8)$ & $(79.8,-, 79.8,79.8)$ \\
$1=4.1 .05-06$ & $(70.0,7.4,75.2,64.7)$ & $(70.0,7.4,75.3,64.8)$ & $(70.1,7.4,75.3,64.8)$ \\
$2=4.1 .06-07$ & $(70.3,5.9,77.7,63.7)$ & $(70.6,5.9,78.0,64.0)$ & $(70.9,5.8,78.3,64.4)$ \\
$3=4.1 .07-08$ & $(71.1,5.4,80.0,64.4)$ & $(71.7,5.4,80.6,65.0)$ & $(72.6,5.5,81.6,65.9)$ \\
$4=4.1 .08-09$ & $(71.7,5.8,81.6,63.5)$ & $(72.7,5.6,82.7,64.4)$ & $(74.1,5.8,84.3,65.6)$ \\
\hline \multicolumn{4}{|c|}{} \\
\hline
\end{tabular}

In Tables $5 \mathrm{a}$ and $5 \mathrm{~b}$ we report the second run (capacity depreciation \& $90 \%$ salvage value case) predicted equilibrium price outcomes ( $\$$ MWh for each year). This 
run is particularly important for a comparison of capital investment levels. The lack of any salvage value and depreciation will tend to cause investments to fall toward zero during the last couple of periods, since capacity investments made in those periods cannot recover costs of investments. We observe that when we consider the salvage value and account for (3.3\% yearly) depreciation in capital, market prices on average through periods 1-4 decrease 13\% (relative to no salvage - no depreciation) with 3-player market and they decrease $8 \%$ with the 5-player market. When number of players increased from three to five, yearly average market price reduced $25 \%$ and yearly average price volatility decreased $31 \%$ in this run.

Table 5a. Yearly price levels with 3 players (90\% salvage value \& depreciation)

\begin{tabular}{|c|c|c|c|}
\hline \multicolumn{3}{|c|}{ Demand Growth } \\
\hline$\underline{\text { Period }}$ & Low $(0.6 \%)$ & Medium $(0.9 \%)$ & High $(1.3 \%)$ \\
\hline $0=4.1 .04-05$ & $(114.8,-, 114.8,114.8)$ & $(114.8,-, 114.8,114.8)$ & $(114.8,-, 114.8,114.8)$ \\
$1=4.1 .05-06$ & $(83.8,8.4,89.7,77.8)$ & $(83.9,8.3,89.8,77.9)$ & $(84.0,8.2,89.8,78.2)$ \\
$2=4.1 .06-07$ & $(83.6,5.0,89.9,77.8)$ & $(84.0,4.9,90.3,78.2)$ & $(84.6,4.9,90.8,78.8)$ \\
$3=4.1 .07-08$ & $(84.7,4.3,91.3,78.2)$ & $(85.4,4.3,91.9,79.0)$ & $(86.4,4.2,92.8,80.0)$ \\
$4=4.1 .08-09$ & $(87.2,4.2,94.1,79.5)$ & $(88.3,4.1,95.0,80.9)$ & $(89.6,3.9,96.3,82.8)$ \\
\hline \multicolumn{4}{|c|}{} \\
\hline
\end{tabular}

Table 5b. Yearly price levels with 5 players $(90 \%$ salvage value \& depreciation)

\begin{tabular}{|c|c|c|c|}
\hline \multicolumn{4}{|c|}{ Demand Growth } \\
\hline Period & Low $(0.6 \%)$ & Medium $(0.9 \%)$ & High $(1.3 \%)$ \\
\hline $0=4.1 .04-05$ & $(79.8,-, 79.8,79.8)$ & $(79.8,-, 79.8,79.8)$ & $(79.8,-, 79.8,79.8)$ \\
$1=4.1 .05-06$ & $(64.2,5.7,68.2,60.1)$ & $(64.2,5.6,68.2,60.2)$ & $(64.3,5.5,68.3,60.4)$ \\
$2=4.1 .06-07$ & $(64.0,3.4,68.3,60.0)$ & $(64.3,3.4,68.6,60.3)$ & $(64.6,3.4,70.0,60.7)$ \\
$3=4.1 .07-08$ & $(64.9,2.8,69.5,61.1)$ & $(65.4,2.7,70.1,61.7)$ & $(66.0,2.7,70.8,62.5)$ \\
$4=4.1 .08-09$ & $(66.9,3.2,72.6,61.3)$ & $(67.7,3.2,73.3,62.2)$ & $(68.8,3.2,74.3,63.5)$ \\
\hline \multicolumn{4}{|c|}{ (Expected price, standard deviation, max price, min price) } \\
\hline
\end{tabular}

In Figure 4, we draw the price evolution for three scenarios (out of sixteen) for the medium demand growth and 90\% salvage value and capital depreciation for the 3-player market. In the figure, s1 refers to $\{$ up, up, up, up\} scenario, s3 refers to \{down, down, down, down $\}$ scenario, s2 refers to $\{$ up, down, down, down $\}$ scenario. 


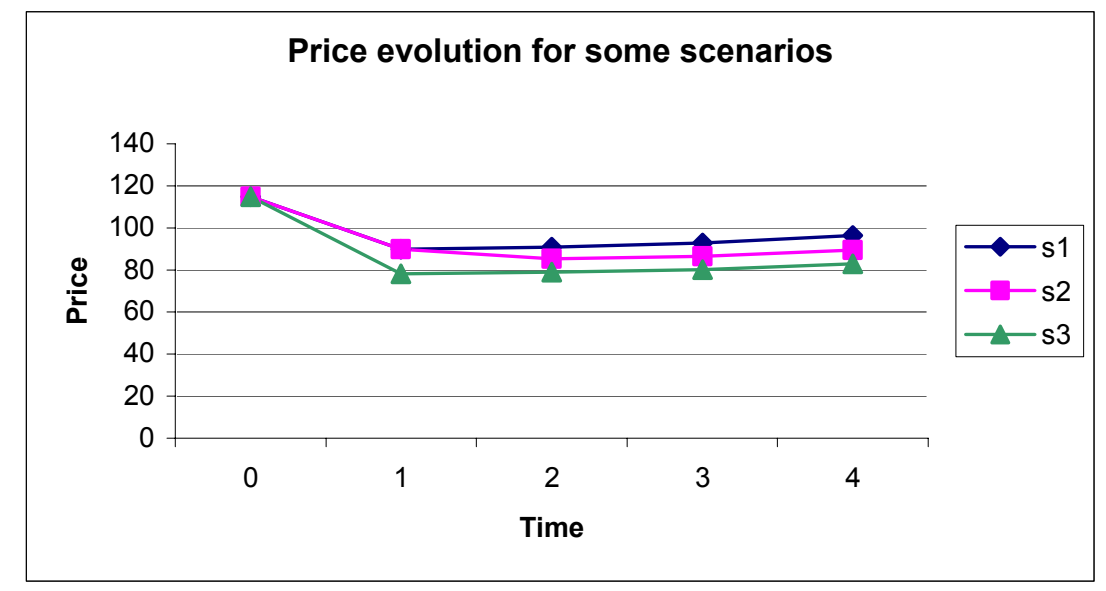

Figure 4: Some price trajectories $(\mathbf{\$} / \mathbf{M W h})$ for $(n, \rho, v)=(3,0.009,0.9)$.

In Figure 5, we present histogram of the equilibrium prices for period 4, with 5players, medium demand growth, yearly capacity depreciation rate $1 / 30$, and salvage value of $90 \%$ of installment cost, based on an interval width equal to $\$ 4 / \mathrm{MWh}$ yearly price.

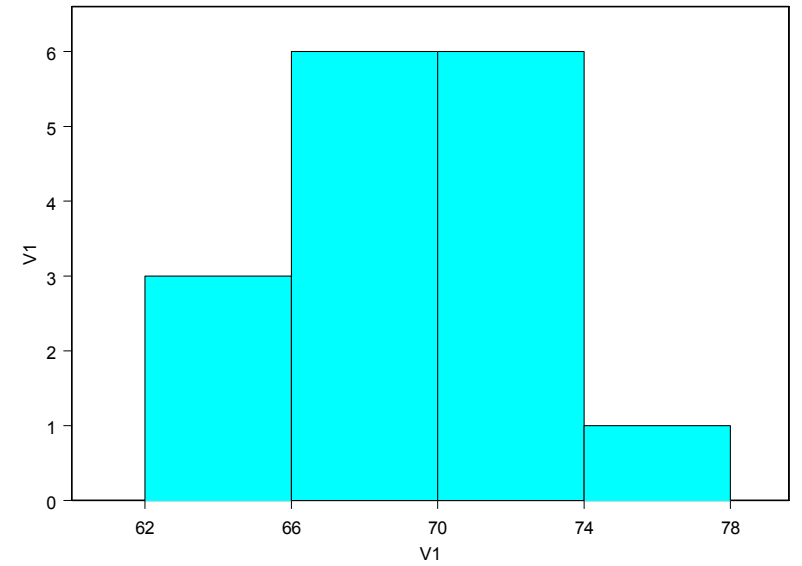

Figure 5: Histogram of equilibrium prices for $(n, t, \rho, v)=(5,4,0.009,0.9)$

\subsection{Connections with other approaches}

A potential alternative to computation of the S-adapted open-loop equilibrium via stochastic programming is to compute a feedback equilibrium using Dynamic Programming. A natural state vector for such an exercise would be the demand state and the vector of capacities for the technologies for each of the players. For the sake of 
definiteness in this discussion, consider the application of our model to the Ontario Power Market, in section 3.3. For the 5-player model, 4 of the players each have one production technology and the fifth player has 3 technologies. This yields a 7-dimensional capacity vector and an 8-dimensional state vector. If this game could be expressed as a finite timehorizon, linear-quadratic (LQ) game then well-known recursive algorithms for computation of linear feedback equilibrium decision rules could be applied (e.g., see Kydland and Prescott (1977) and also Reynolds (1986)). However, an LQ formulation does not permit constraints on decisions (e.g., production bounded below by zero and above by capacity) nor does it provide a good approximation of capacity constrained costs. A quadratic payoff would imply that marginal production cost is linear in output for all output levels. This is a bad approximation of an increasing marginal cost curve that becomes vertical at output equal to capacity.

Another approach would be to discretize the state space by restricting capacity levels to belong to a finite set of $r$ numbers. The DP approach would involve computation of a feedback equilibrium value function for each player for each possible state. The number of possible states in period $t$ for the Power Market application is $2^{t} r^{7}\left(2^{t}\right.$ is the number of demand states in $t, r^{7}$ is the number possible capacity vectors). For example, if $r=12$ then there are over 570 million states in the final period ( $t=4)$, over 285 million possible states in the penultimate period $(t=3)$, and so on. ${ }^{3}$ This illustrates the curse of dimensionality for a DP approach. Application of the DP approach to this seemingly modest-sized problem turns out to involve severe computational difficulties.

In defense of DP however, we should note that it allows one to conceptualize a richer set of strategies, namely, equilibrium in feedback strategies. The economics literature refers to this as a Markov perfect equilibrium (e.g., Ericson and Pakes (1995), Lockwood (1996)) or a subgame perfect equilibrium. A feedback strategy is conditioned not only on the time period and demand state, but also on a state vector that summarizes

\footnotetext{
${ }^{3}$ Of course, if $r$ is smaller the dimensionality is reduced. But a courser grid of capacity values may be an inferior approximation of the true state space. A discrete state space may also introduce new problems of multiple equilibria. Berry and Pakes (1993) utilize a probabilistic investment formulation that smoothes out discreteness for investment choices and avoids the multiple equilibria problem. However, this formulation does not eliminate the "curse of dimensionality".
} 
the historical decisions that are relevant for players' current and future payoffs. ${ }^{4}$ Such a formulation allows one to consider incentives for strategic investment, since the investment decisions of any one player may influence later decisions of other players (see Reynolds $(1986,1987,1991)$ for analyses of this type). Note that strategic incentives of this type are absent in the open-loop formulation. Reynolds (1987) shows that the quantitative difference between feedback equilibria and open-loop equilibria is relatively small for simple dynamic models of production and investment. ${ }^{5}$ Moreover to date, equilibria of this type have not been computed for large-scale models, although as demonstrated in this paper, S-adapted open loop policies are computable using stochastic programming.

For reasons outlined above, we do not compute a subgame perfect equilibrium. However, results in this paper and prior papers suggest how subgame perfect equilibrium results would compare to results for the GES and GPS formulations of this paper.We show in Example 1 that a player's payoff can be higher in a GES formulation than in a GPS (equivalently, open loop) formulation Next, Reynolds $(1986,1987)$ shows for deterministic investment and production games, that players' payoffs are lower in the subgame perfect equilibrium than in the open loop equilibrium. The reason is that a subgame perfect equilibrium introduces an additional strategic incentive that is not present in the open loop (or, GES) formulation; in a subgame perfect equilibrium, each player recognizes that extra investment now will preempt some future investment by rivals. These incentives lead to greater investment and production overall, and lower prices, in a subgame perfect equilibrium than in an open loop equilibrium. This strategic incentive to invest should also be present in a subgame perfect equilibrium formulation with demand uncertainty. These two comparisons, namely GES vs. open loop and open loop vs. subgame perfect, suggest that payoffs would be lower in a subgame perfect equilibrium than in a GES solution.

\footnotetext{
${ }^{4}$ The concept of subgame perfect equilibrium is more general than feedback equilibrium. The set of subgame perfect equilibria may include equilibria in which firms behave collusively; collusion is enforced by threats of reversion to competitive behavior if deviation from collusion is observed.

${ }_{5}^{5}$ Also, see Deneckere and de Palma (1998) for a defense of using the open-loop concept in dynamic games.
} 


\section{Conclusions}

In this paper we have studied several alternative games under uncertainty. These include Games with Probabilistic Scenarios (GPS), Games with Expected Scenarios (GES), and a Hybrid Game (HG). The HG formulation provides the argument against GES, even though there are instances in which all players may be better off by choosing to play the GES over GPS. Thus under uncertainty, it is plausible to consider the GPS formulation. We also show that if all players are identical, and the probability of a higher inverse-demand curve either equals or exceeds that of a lower inverse-demand curve, then players can earn greater expected profits as volatility increases. This result suggests that even in an increasingly volatile market, players may have an incentive to participate in the market. Given the surge in uncertainty within certain markets (e.g., energy), this result appears to be reassuring.

Our approach, based on stochastic programming, provides a computationally realistic approach to oligopolistic games under uncertainty. The key to this presentation is the so-called scenario formulation which allows us to model the games through a finite number of scenarios, coordinated by the non-anticipativity constraints. The same type of formulation holds for both two-stage, as well as multi-stage games, both of which are solvable using standard algorithms for linear complementarity problems (e.g. the PATH solver available on the NEOS server). The Ontario power market application presented in this paper illustrates that this approach can address dynamic games that are clearly out of reach for dynamic programming, a common approach in the literature on dynamic games. The theory associated with infinitely-many scenarios within the stochastic programming setting is currently under investigation, and some initial results are available in Higle and Sen (2004) and Casey and Sen (2004).

Acknowledgements: The authors thank the referees for their comments on previous versions which helped focus the current version. We are also grateful to the editor-inchief for his encouragement during the refereeing process. Finally, the research reported in this paper was supported under a grant from the National Science Foundation: DMI 9978780 and another AFOSR/MURI contract F49620-03-1-0477. 


\section{References}

Berry, S., Pakes, A., 1993. Some Applications and Limitations of Recent Advances in Empirical Industrial Organization: Merger Analysis. American Economic Review 83.

Birge, J.R., Louveaux, F., 1997. Introduction to Stochastic Programming, Springer.

Branch, E.R., 1993. Short run income elasticity of demand for residential electricity using consumer expenditure survey data. Energy Journal 14.

Casey, M., Sen, S., 2004. "The Scenario Generation Algorithm for Multi-stage Stochastic Linear Programming” forthcoming in Mathematics of Operations Research.

Deneckere, R. J., de Palma, A., 1998. The diffusion of consumer durables in a vertically differentiated oligopoly. Rand Journal of Economics 29.

Dixit, A., Pindyk, R., 1997. Investment under uncertainty. Princeton University Press, Princeton, New Jersey.

Energy information administration, http://www.eia.doe.gov/

Ericson, R., Pakes, A., 1995. Markov Perfect Industry Dynamics: A framework for Empirical Work. Review of Economic Studies 62.

Friedman, J.M., 1977. Oligopoly Theory. North Holland.

Forgo, F., Szidarovsky, F., 1999. Introduction to the Theory of Games. Kluwer Academic Publishers.

Garcia-Diaz, A., Marin, P.L., 2003. Strategic bidding in electricity pools with short-lived bids: an application to the Spanish market. International Journal of Industrial Organization 21.

Green, R.J., Newbery, D.W., 1992. Competition in the British electricity spot market. Journal of Political Economy 100.

Gurkan, G., Ozge, A.Y., Robinson, S.M., 1999. Sample-path solution of stochastic variational inequalities. Mathematical Programming 84, 313-333.

Haurie, A., Zaccour, G., Legrand, J., Smeers, Y., 1987. A stochastic dynamic NashCournot model for the European gas market. Technical Report G-87-24. GERAD, Ecole des Hautes Etudes Commerciales, Montréal, Quebec, Canada.

Haurie, A., Zaccour, G., Smeers, Y., 1990. Stochastic Equilibrium Programming for Dynamic Oligopolistic Markets. Journal of Optimization Theory and Applications 66. 
Haurie, A., Roche, M., 1994. Turnpikes and computation of piecewise open-loop equilibria in stochastic differential games. Journal of Economic Dynamics and Control $18,317-344$.

Haurie, A., Moresino, F., 2001. Computation of S-adapted equilibrium in piecewise deterministic games via stochastic programming methods. In Advances in Dynamic Games and Applications Eitan Alman and Odile Pourtallier eds., Annals of the International Society of Dynamic Games 6, Birkhauser Boston, ISBN 0-8176-4202, 37643-4202.

Haurie, A., Moresino, F., 2002. S-adapted oligopoly equilibria and approximations in stochastic variational inequalities. Annals of Operations Research 114, 183-201.

Haurie, A., Viguier, L., 2003. A Stochastic dynamic game of carbon emissions trading. Environmental Modeling \& Assessment 8, 239-248.

Haurie, A., Zaccour, G., 2004. S-adapted equilibria in games played over event trees: an overview. Annals of the International Society of Dynamic Games 7, 367-400.

Higle, J.L., Sen. S., 2004. Duality for Multistage Convex Stochastic Programs. Forthcoming in Annals of Operations Research.

Konnov, I., 1993. Combined relation methods for finding equilibrium points and solving related problems. Izvestiya VUZ, Mathematika 37, 44-51.

Kydland, F., Prescott, E., 1977. Rules Rather Than Discretion : The Inconsistency of Optimal Plans. Journal of Political Economy 85.

Lockwood, B., 1996. Uniqueness of Markov-perfect equilibrium in infinite-time affinequadratic differential games. Journal of Economic Dynamics and Control 20.

Murphy, F.H., Sen, S., Soyster, A., 1982. Electric Utility Capacity Expansion Planning with Uncertain Load Forecasts. AIIE Transactions 14.

Mussa, M., 1977. External and Internal Adjustment Costs and the Theory of Aggregate and Firm Investment. Economica 44.

National energy board, http://www.neb-one.gc.ca/

NEOS Server, www-neos.mcs.anl.gov

Okuguchi, K., Szidarovszky, F., 1999. The Theory of Oligopoly with Multi-Product Firms. New York: Springer Verlag.

Ontario power generation, www.opg.com. 
Pakes, A., McGuire, P., 1994. Computing Markov-Perfect Nash Equilibria: Numerical Implications of a Dynamic Differentiated Product Model. Rand Journal of Economics 25.

Reynolds, S., 1986. Strategic Capital Investment In the American Aluminum Industry. The Journal of Industrial Economics 34.

Reynolds, S., 1987. Capacity Investment, Preemption and Commitment in an Infinite Horizon Model. International Economic Review 28.

Reynolds, S., 1991.Dynamic Oligopoly with Capacity Adjustment Costs. Journal of Economic Dynamics and Control 15.

The independent market operator, www.theimo.com

Taylor, L.D., 1975. The demand for electricity: a survey. Rand Journal of Economics 6.

Taylor, J.J., 2001. Economic and market potential of small innovative reactors. Mimeo, The James A.Baker III Institute for Public Policy of Rice University.

WASP-IV, 2000. Wein Automatic System Planning. Version IV, International Atomic Energy Commission.

Wolfram, C.D., 1999. Measuring duopoly power in the British electricity spot market. American Economic Review 89.

Zaccour, G., 1987. Théorie des jeux et marchés énergétiques: marché européén de gaz naturel et échanges d'électricité. PhD thesis, HEC, Montreal. 